\title{
Realistic Two-body Interactions in Many-nucleon Systems: Correlated Motion beyond Single-particle Behavior
}

\author{
K. D. Sviratcheva, ${ }^{1}$ J. P. Draayer ${ }^{1}$ and J. P. Vary ${ }^{2}$ \\ ${ }^{1}$ Department of Physics and Astronomy, Louisiana State University, Baton Rouge, Louisiana 70803, USA \\ ${ }^{2}$ Department of Physics and Astronomy, Iowa State University, Ames, IA 50011, USA \\ and \\ Lawrence Livermore National Laboratory, Livermore, California 94551 \\ and \\ Stanford Linear Accelerator Center, Stanford University, Stanford, California 94309
}

(Dated: June 13, 2006)

\begin{abstract}
In the framework of the theory of spectral distributions we perform an overall comparison of three modern realistic interactions, CD-Bonn, CD-Bonn+3terms, and GXPF1 in a broad range of nuclei in the upper $f p$ shell and study their ability to account for the development of isovector pairing correlations and collective rotational motion in many-particle nuclear systems. Our findings reveal a close similarity between CD-Bonn and CD-Bonn+3terms, while both interactions possess features different from the ones of GXPF1. The GXPF1 interaction is used to determine the strength parameter of a quadrupole term that augments an isovector-pairing model interaction with $\mathrm{Sp}(4)$ dynamical symmetry, which in turn is shown to yield a reasonable agreement with the experimental low-lying energy spectra of ${ }^{58} \mathrm{Ni}$ and ${ }^{58} \mathrm{Cu}$.
\end{abstract}

\section{INTRODUCTION}

Realistic $N N$ potentials, whether derived from meson exchange theory (e.g.,[1]) or chiral effective field theory (e.g., [2]), provide no clear indication until utilized by shell-model calculations on how well they can reproduce prominent features of nuclei such as pairing gaps in nuclear spectra or enhanced electric quadrupole transitions in collective rotational bands. While the properties of a shell-model many-body wavefunction are usually affected by the adopted model, a direct examination of a realistic interaction based on the spectral distribution theory $[3,4]$ can bring forward a deeper understanding of the very nature of the interaction and above all, its role in the development of collective and correlated many-nucleon motion [5-9]. In addition it can reveal its similarity, via correlation coefficients, to other interactions including the pairing and quadrupole-quadrupole interactions. Such analysis is independent of the averages of the interactions and yields an overall comparison throughout a broad domain of nuclei beyond what can be achieved by overlaps of several nuclear states or matching a group of two-body interaction matrix elements.

In the paper we compare three modern realistic interactions, namely, the CD-Bonn [1], CD-Bonn+3terms [10] and GXPF1 [11], as well as two model interactions with pairing and quadrupole terms, which typically dominate in nuclei. The present study, which is complementary to a similar $1 f_{7 / 2}$ analysis [12], focuses on the upper $f p$-shell domain. Such a partitioning of the $f p$ oscillator shell follows naturally from a splitting of these two regions by a strong spin-orbit interaction. The rotational and pairing characteristics of a realistic interaction can be probed through its projection onto a microscopic model Hamiltonian that describes collective rotational excitations or pairing correlations [6, 13-15]. If the model and realistic interactions are strongly correlated, then the latter will reflect the characteristic properties of the quadrupole (pairing) Hamiltonian, which in turn may be used as a good approximation.

Within the framework of the harmonic oscillator shellmodel, the quadrupole and pairing two-body interactions possess a clear algebraic structure. While in the quadrupole limit the $\mathrm{SU}(3)$ symmetry [16] governs a shape-determined dynamics, the like-particle and protonneutron (isovector) pairing correlations respect a $\operatorname{Sp}(4)$ dynamical symmetry [17-19]. Specifically, we employ the $\mathrm{Sp}(4)$ model interaction [20, 21], which has been found to provide for a reasonable microscopic description of the pairing-governed isobaric analog $0^{+}$states in light and medium mass nuclei [21, 22], such as neutron-deficient and $N \approx Z$ nuclei along the nucleosynthesis $r p$-path and unstable nuclei currently explored in radioactive beam experiments $[23,24]$. In addition, the $\mathrm{Sp}(4)$ model interaction has been shown to account quite well for the observed detailed structure beyond mean-field effects such as the $N=Z$ anomalies, isovector pairing gaps and staggering effects [22] and as well to strongly correlate, especially when the quadrupole term is introduced, with the realistic interactions under consideration in the $1 f_{7 / 2}$ orbit [12].

Several detailed reviews of the nuclear shell model and its applications have been published recently [25-27] that delve into related key physics issues that are explored in the present work. However, the spectral distribution analysis provided here is novel and sheds considerable light on the features of new $f p$-shell interactions, some of which have been developed since those reviews were completed. 


\section{THEORETICAL FRAMEWORK}

\section{A. Theory of Spectral Distributions}

The theory of spectral distributions is an excellent approach for studying microscopic interactions [4, 28, 29] and continues to be a powerful concept with recent applications in quantum chaos, nuclear reactions and nuclear astrophysics including studies on nuclear level densities, transition strength densities, and parity/time-reversal violation (for example, see [30-39]). The significance of the method is related to the fact that low-order energy moments over a certain domain of single-particle states, such as the energy centroid of an interaction (its average expectation value) and the deviation from that average, yield valuable information about the interaction that is of fundamental importance [6, 15, 29, 40-45] without the need for carrying out large-dimensional matrix diagonalization and with little to no limitations due to the dimensionality of the vector space.

Spectral distribution theory (see Appendix for basic mathematical definitions and notation introduction) combines important features, the most significant of which are as follows:

1. The theory provides a precise measure, namely, the correlation coefficient, for the overall similarity of two interactions. Literally the correlation coefficient is a measure of the extent to which two interactions "look like" (are correlated with) one another. In this respect, correlation coefficients can be used to extract information how well pairing/rotational features are developed in realistic interactions, which may differ substantially from an individual comparison of pairing/quadrupole interaction strengths [12].

2. It gives an exact prescription for identifying the pure zero- (centroid), one- and two-body parts of an interaction under a given space partitioning. Therefore, major properties follow:

(a) The correlation coefficients are independent of the interaction centroids. (A direct comparison of two-body matrix elements provided by $N N$ potentials may be misleading, especially when the averages of the interactions differ considerably.)

(b) The pure one-body part extracted from the interaction, the so-called induced single-particle energies (Appendix Eq. 17), is the average monopole interaction (compare to [11]) and as such influences the evolution of the shell structure, shell gaps and binding energies [46].

(c) The pure two-body part is essential for studies of detailed property-defining two-body interactions beyond strong mean-field effects.
3. The correlation coefficient concept can be propagated straightforwardly beyond the defining twonucleon system to derivative systems with larger numbers of nucleons [4] and higher values of isospin [5]. This, in addition to the two-nucleon information provided by alternative approaches (e.g., [47]), yields valuable overall information, without a need for carrying out extensive shell-model calculations, about the universal properties of a two-body interaction in shaping many-particle nuclear systems.

Group theory underpins spectral distribution theory $[3-5,7,48]$. The model space is partitioned according to particular group symmetries and each subsequent subgroup partitioning yields finer and more detailed spectral estimates. Specifically, for $n$ particles distributed over $\mathcal{N}$ single-particle states, the spectral distribution averaged over all $n$-particle states associated with the $\mathrm{U}(\mathcal{N}=4 \Omega)$ group structure is called a scalar distribution (denoted by " $n$ " in the formulae) and the spectral distribution averaged over the ensemble of all $n$-particle states of isospin $T$ associated with $\mathrm{U}(\mathcal{N}=2 \Omega) \otimes \mathrm{U}(2)_{T}$ is called an isospinscalar distribution (denoted by " $n, T$ ").

For a spectral distribution $\alpha(\alpha$ is $n$ or $n, T)$, the correlation coefficient between two Hamiltonian operators, $H$ and $H^{\prime}$, is defined as

$$
\begin{aligned}
\zeta_{H, H^{\prime}}^{\alpha} & =\frac{\left\langle\left(H^{\dagger}-\left\langle H^{\dagger}\right\rangle^{\alpha}\right)\left(H^{\prime}-\left\langle H^{\prime}\right\rangle^{\alpha}\right)\right\rangle^{\alpha}}{\sigma_{H} \sigma_{H^{\prime}}} \\
& =\frac{\left\langle H^{\dagger} H^{\prime}\right\rangle^{\alpha}-\left\langle H^{\dagger}\right\rangle^{\alpha}\left\langle H^{\prime}\right\rangle^{\alpha}}{\sigma_{H} \sigma_{H^{\prime}}}
\end{aligned}
$$

where the "width" of the distribution is the positive square root of the variance,

$$
\left(\sigma_{H}^{\alpha}\right)^{2}=\left\langle\left(H-\langle H\rangle^{\alpha}\right)^{2}\right\rangle^{\alpha}=\left\langle H^{2}\right\rangle^{\alpha}-\left(\langle H\rangle^{\alpha}\right)^{2},
$$

and the steps for computing these quantities are outlined in the Appendix. The average values, $\langle H\rangle^{\alpha}$, related to the trace of an operator divided by the dimensionality of the space, are given in terms of the ensemble considered. In the (isospin-)scalar case, the correlation will be denoted by $\zeta^{n}\left(\zeta^{n, T}\right)$ or simply $\zeta\left(\zeta^{T}\right)$ for $n=2$.

From a geometrical perspective, in spectral distribution theory every interaction is associated with a vector and the correlation coefficient $\zeta$ (Eq. 1) defines the angle (via a normalized scalar product) between two vectors of length $\sigma$ (Eq. 2). Hence, $\zeta_{H, H^{\prime}}$ gives the normalized projection of $H$ onto the $H^{\prime}$ interaction (or $H^{\prime}$ onto $H$ ). In addition, $\left(\zeta_{H, H^{\prime}}\right)^{2}$ gives the percentage of $H$ that reflects the characteristic properties of the $H^{\prime}$ interaction.

\section{B. Description of Interactions}

Here we examine three modern realistic interactions (which will be denoted as $H_{R}$ ), particularly two CDBonn interactions [1, 10] and GXPF1 [11]. CD-Bonn is a charge-dependent one-boson-exchange nucleon-nucleon 
$(N N)$ potential that is one of the most accurate in reproducing the available proton-proton and neutron-proton scattering data. We employ the two-body matrix elements of the effective interaction derived from CDBonn for $0 \hbar \omega$ no-core shell model (NCSM) calculations in the $f p$ shell. In addition, the CD-Bonn+3terms interaction introduces phenomenological isospin-dependent central terms plus a tensor force with strengths and ranges determined in no-core $0 \hbar \omega$ shell model calculations to achieve an improved description of the $A=48$ $\mathrm{Ca}, \mathrm{Sc}$ and $\mathrm{Ti}$ isobars. The GXPF1 effective interaction is obtained from a realistic G-matrix interaction based on the Bonn-C potential [49] by adding empirical corrections determined through systematic fitting to experimental energy data in the $f p$ shell.

The model interactions include a $\mathrm{Sp}(4)$ Hamiltonian with isosvector pairing plus $p n$ isoscalar force and an extended pairing+quadrupole interaction. The general rotationally invariant model Hamiltonian with $\mathrm{Sp}(4)$ dynamical symmetry for a system of $n$ valence nucleons in a $4 \Omega$-dimensional space that conserves the number of particles $\left(\left[H_{\mathfrak{s p}(4)}, \hat{N}\right]=0\right)$ and the third projection of the isospin $\left(\left[H_{\mathfrak{s p}(4)}, \hat{T}_{0}\right]=0\right)$ can be expressed through the $\mathrm{Sp}(4)$ group generators,

$$
\begin{aligned}
& H_{\mathfrak{s p}(4)}=-G \sum_{i=-1}^{1} \hat{A}_{i}^{\dagger} \hat{A}_{i}-F \hat{A}_{0}^{\dagger} \hat{A}_{0}-\frac{E}{2 \Omega}\left(\hat{T}^{2}-\frac{3 \hat{N}}{4}\right) \\
& -D\left(\hat{T}_{0}^{2}-\frac{\hat{N}}{4}\right)-C \frac{\hat{N}(\hat{N}-1)}{2}-\epsilon \hat{N},
\end{aligned}
$$

with two-body antisymmetric JT-coupled matrix elements (Eq. 7) for isospin rank 0 and 2 of $H_{\mathfrak{s p}(4)}$ and $\{r \leq(s, t) ; t \leq u\}$ orbits,

$$
\begin{aligned}
W_{r s t u}^{(0) J T} & \equiv W_{r s t u}^{J T}=<r s J T M T_{0}\left|H^{(0)}\right| t u J T M T_{0}> \\
& =-G_{0} \frac{\sqrt{\Omega_{r} \Omega_{t}}}{\Omega} \delta_{(J T),(01)} \delta_{r s} \delta_{t u} \\
& -\left\{-E_{0}\left[(-)^{T}+\frac{1}{2}\right]+C\right\} \delta_{r t} \delta_{s u} \\
W_{r s t u}^{(2) J T} & =<r s J T M T_{0}\left|H^{(2)}\right| t u J T M T_{0}> \\
& =\frac{\sqrt{2}}{3}\left(F \frac{\sqrt{\Omega_{r} \Omega_{t}}}{\Omega} \delta_{J 0} \delta_{r s} \delta_{t u}-D \delta_{r t} \delta_{s u}\right) \delta_{T 1}
\end{aligned}
$$

where $G_{0}=G+\frac{F}{3}, E_{0}=\left(\frac{E}{2 \Omega}+\frac{D}{3}\right), G, F, E, D$ and $C$ are interaction strength parameters and $\epsilon>0$ is the Fermi level energy (see Table I in Ref.[21] for parameter estimates). The $\mathfrak{s p}(4)$ algebraic structure is exactly the one needed in nuclear isobaric analog $0^{+}$states to describe proton-proton $(p p)$, neutron-neutron $(n n)$ and protonneutron $(p n)$ isovector pairing correlations, accounted by the pair annihilation (creation) operators $\hat{A}_{+1,-1,0}^{(\dagger)}$, and isospin symmetry, reflected by the isospin operator $\hat{T}^{2}$ and related to a $J$-independent isoscalar $(T=0) p n$ force. While the isotensor part (Eq. 5) of the model interaction lies outside of the scope of the present examination, we mention for completeness that it introduces isospin symmetry breaking through the $D$-term and as well a plausible, but very weak, isospin mixing ( $F$-term) [50] in the Hamiltonian.
Furthermore, higher- $J$ states in the low-lying nuclear energy spectra enter the analysis and these states require the inclusion of an additional quadrupole-quadrupole interaction that being symmetric under SU(3) breaks the $\mathrm{Sp}(4)$ symmetry and lifts degeneracies,

$$
H_{M}=H_{\mathfrak{s p}(4)}+H_{Q}^{\perp}(2), H_{Q}=-\frac{\chi}{2} Q \cdot Q .
$$

The $H_{Q}^{\perp}(2)$-term is the part of the pure two-body $H_{Q}(2)$ interaction that is not contained in $\operatorname{Sp}(4)$. In the vector algebra terminology this means that we add the part that is orthogonal to the pure two-body $\mathrm{Sp}(4)$ Hamiltonian [6]. This is because the $\mathrm{Sp}(4)$ interaction contains a part of the quadrupole-quadrupole interaction. This part is not negligible as revealed by the correlation between $H_{Q}$ and $H_{\mathfrak{s p}(4)}$. Namely, in the scalar case it is $15 \%\left(1 f_{7 / 2}\right), 29 \%$ $\left(1 f_{5 / 2}\right)$ and $29 \%\left(2 p_{1 / 2} 2 p_{3 / 2}\right)$, and for the $\mathrm{T}=1$ part of the interactions in the isospin-scalar case, it is $34 \%\left(1 f_{7 / 2}\right)$, $58 \%\left(1 f_{5 / 2}\right)$ and $58 \%\left(2 p_{1 / 2} 2 p_{3 / 2}\right)$. This is probably one of the reasons why the $\mathrm{Sp}(4)$ model interaction turns out to work rather well despite no explicit appearance of the quadrupole-quadrupole interaction.

Such a Hamiltonian (Eq. 6) does not affect the centroid of $H_{\mathfrak{s p}(4)}$ because $H_{Q}^{\perp}(2)$ is traceless. In this way this collective interaction preserves the shell structure that is built into $H_{\mathfrak{s p}(4)}$ and established by a harmonic oscillator potential and as a result is favored in many studies $[6$, $15,51,52]$.

The interaction matrix elements (Eq. 4) correspond to the pure nuclear interaction and do not include Coulomb repulsion, which, in the $\mathrm{Sp}(4)$ model, is corrected in the experimental energies themselves by applying an empirical formula deduced in [53]. This may result in slightly more bound states predicted by our model when compared to estimates of realistic interactions.

\section{UNDERSTANDING THE NUCLEAR INTERACTION IN MANY-NUCLEON SYSTEMS}

The similarity of different interactions and their pairing/rotational characteristics can be tracked in manynucleon systems [14] through the propagation formulae (Appendix Eqs. 15, 19). The latter determine how the averages extracted from the two-nucleon matrix elements get carried forward into many-nucleon systems. This propagation of information is model-independent.

We examine the pure two-body part of the realistic interactions, $H_{R}(2)$, and how it is correlated to the isospinconserving part of the model interactions under consideration, (3) and (6). The latter, in addition to their centroids, are pure two-body in the upper $f p$ model space because of the assumption for $H_{\mathfrak{s p}(4)}$ of constant Fermi level energy and fixed interaction strengths throughout the entire region. The significance of the correlation coefficients between pure two-body interactions [29] reflect the fact that nuclear states, their collective properties and configuration mixing, are solely shaped by the pure 
two-body part of an interaction, while the one-body part, albeit of a considerable significance, trivially reorders the states in the nuclear energy spectrum. In addition, such analyses are free of the one-body influence including induced single-particle energies, which are related to the monopole interaction $[11,46,47]$, and external singleparticle energies. The latter are introduced when a core is assumed, as for the $0 \hbar \omega{ }^{40} \mathrm{Ca}$-core shell model using the GXPF1 interaction, but not for the $0 \hbar \omega$ no-core shell model with the CD-Bonn or CD-Bonn+3terms interactions. For the NCSM interactions, the two-body matrix elements specifying the particle-core interactions supplant the role of the external single-particle energies included with the GXPF1 interaction. These additional two-body matrix elements together with the external single-particle energies for GXPF1 are not included in the present analyses.

In our study, we vary only $\chi$, the quadrupole strength parameter in (Eq. 6), to find its optimal value (which is an exact solution) by maximizing the $\zeta$ correlation coefficient [54] between the model $H_{M}$ interaction and the pure two-body part $H_{R}(2)$ of each of the realistic interactions under consideration. We do not alter the parameters of the $\mathrm{Sp}(4)$ model, which have already been shown in an appropriate domain of states to be valid for reproducing various quantities (such as binding energies and pairing gaps) and are in agreement with estimates available in literature [21, 22].

In both scalar (Table I) and isospin-scalar (Fig. 1) distributions, the $\zeta_{H_{R}(2), H_{M}}^{n(T)}$ correlation of the realistic interactions with the pairing+quadrupole extended model interaction is stronger for the GXPF1 interaction compared to CD-Bonn and CD-Bonn+3terms $\left(\zeta_{G X P F 1(2), H_{M}} \approx 0.8\right.$ is much higher than 0.6 typically regarded as good [8]). Hence, other types of interactions that do not correlate with the pairing and quadrupole-quadrupole interactions (of fixed strength throughout the upper $f p$ shell) comprise a relatively small part of the pure two-body GXPF1 interaction. They are weakest for the $T=n / 2$ group of states (Fig. 1) for all the three interactions.

In this isospin-scalar space partitioning (Fig. 1), where the ability of a realistic interaction to form correlated pairs is detected via $\zeta_{H_{R}(2), H_{\mathfrak{s p}(4)}, T}$, which is independent of the pairing strength parameters in $H_{\mathfrak{s p}(4)}(\mathrm{Eq}$. 3), the $T=n / 2$ group of states is where the $\operatorname{Sp}(4)$ symmetry and particularly the isovector pairing correlations are manifested. This includes the $T=1$ part of the interaction itself $(n=2)$. The results show a good tendency in GXPF1 for development of pairing correlations in the $T=n / 2$ states as was the case for the $1 f_{7 / 2}$ domain. For the three interactions, the additional quadrupolequadrupole interaction, $H_{Q}^{\perp}(2)$, is comparatively (much) smaller for the highest-isospin states, while it increases towards low-isospin states.

Compared to the pairing $H_{\mathfrak{s p}(4)}$ Hamiltonian, the additional collective interaction, $H_{Q}^{\perp}(2)$, that is the part of
TABLE I: Correlation coefficients for many-nucleon systems of the pure two-body realistic interactions $H_{R}(2)$ with $H_{\mathfrak{s p}(4)}$ and $H_{Q}^{\perp}(2)$, with the extended pairing+quadrupole model interaction $H_{M}($ Eq. 6), and with the pure twobody full quadrupole-quadrupole interaction, $H_{Q}(2)$. The $\left(\zeta_{H_{M}, H_{\mathfrak{s p}(4)}}\right)^{2}$ quantity gives the part, in $\%$, of $H_{M}$ that is $\mathrm{Sp}(4)$ symmetric.

\begin{tabular}{lccc}
\hline & CD-Bonn & CD-Bonn+3terms & GXPF1 \\
\hline \hline$\zeta_{H_{R}(2), H_{\mathfrak{s p}(4)}}$ & 0.55 & 0.50 & 0.65 \\
$\zeta_{H_{R}(2), H_{Q}^{\perp}(2)}$ & 0.14 & 0.20 & 0.51 \\
$\zeta_{H_{R}(2), H_{M}}$ & 0.57 & 0.54 & 0.83 \\
$\left(\zeta_{H_{M}, H_{\mathfrak{s p}(4)}}\right)^{2}$ & $93.1 \%$ & $85.7 \%$ & $61.3 \%$ \\
$\zeta_{H_{R}(2), H_{Q}(2)}$ & 0.28 & 0.33 & 0.67 \\
\hline
\end{tabular}

the $H_{Q}(2)$ rotational interaction that is not included in $H_{\mathfrak{s p}(4)}$, has a lower correlation with $H_{R}$ for all the realistic interactions in the upper $f p$ (Table I, second row and Fig. 1) except $T \neq n / 2$ for GXPF1 in the isospin-scalar case. This result implies that a comparatively larger part of the overall correlations is already accounted for solely by the model interaction with symplectic $\mathrm{Sp}(4)$ dynamical symmetry, which, especially for both CD-Bonn interactions, is only slightly broken by $H_{Q}^{\perp}(2)$ (Table I, fourth row and Fig. 1). The $\operatorname{Sp}(4)$ symmetry breaking is related to the correlation coefficient of $H_{Q}^{\perp}(2)$ with $H_{M}$ or equivalently to the ratio of their norms [55], where $\left(\zeta_{H_{M}, H_{Q}^{\perp}(2)}\right)^{2}=1-\left(\zeta_{H_{M}, H_{\mathfrak{s p}(4)}}\right)^{2}$ (Table I).

The capability of a realistic interaction to describe rotational collective motion, and hence to reproduce rotational bands and enhanced electric quadrupole transitions, can be detected via its correlation to the full $H_{Q}(2)$ quadrupole-quadrupole two-body interaction (Table I, fifth row and Fig. 2). In both scalar and isospinscalar cases, the rotational features are more fully developed for GXPF1 and less for CD-Bonn+3terms and CDBonn (Fig. 2) different from the outcome in the $1 f_{7 / 2}$ region especially for the $T=1$ part of CD-Bonn. As expected, compared to the orthogonal $H_{Q}^{\perp}(2)$ interaction in (Eq. 6), the $\zeta_{H_{R}(2), H_{Q}(2)}$ correlations are much higher. In the $T=n / 2$ group of states the full quadrupolequadrupole contribution is the strongest (weakest) for CD-Bonn+3terms (CD-Bonn and GXPF1).

In short, the GXPF1 interaction is expected to reproduce spectral features like pairing gaps and rotational bands observed in the upper $f p$ nuclei, while it is unlikely for both CD-Bonn interactions to fully reflect the rotational properties of these nuclei. In comparison, the CD-Bonn+3terms interaction in the $1 f_{7 / 2}$ orbit exhibits well-developed pairing and rotational characteristics [12] and hence it is expected to achieve a rather good description of the associated many-body phenomena in the energy spectra of nuclei with valence nucleons occupying the $1 f_{7 / 2}$ orbit. Such a difference in the behavior of CDBonn+3terms within both regions, $1 f_{7 / 2}$ and upper $f p$, may reflect the fact that this interaction was deter- 


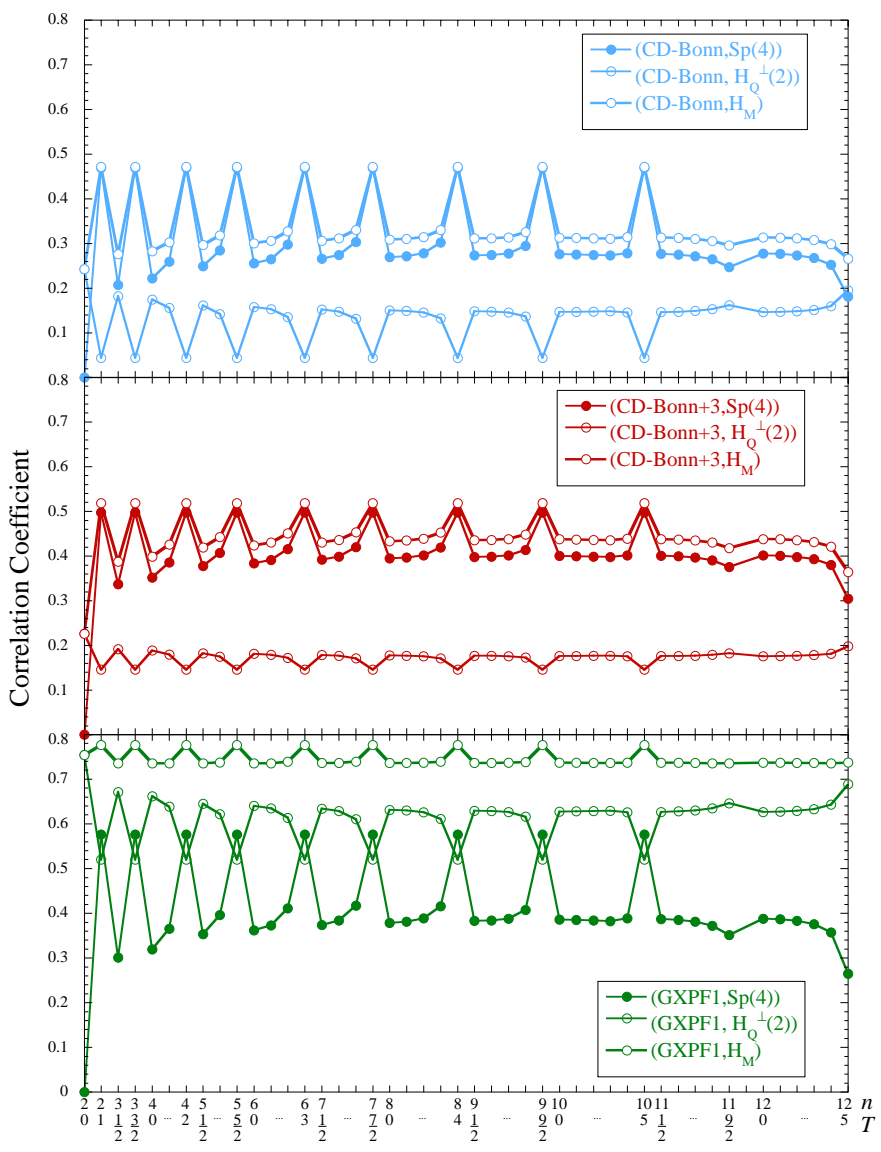

FIG. 1: Correlation coefficients of the pure two-body realistic interactions, CD-Bonn (blue), CD-Bonn+3terms (red) and GXPF1 (green), with $H_{\mathfrak{s p}(4)}$ (filled symbols), $H_{Q}^{\perp}(2)$ (transparent symbols) and $H_{M}$ (empty symbols) in the upper $f p$ shell for the isospin-scalar distribution. For each valence particle number, $n$, the isospin $T$ varies as $\frac{n}{2}, \frac{n}{2}-1, \ldots, 0(1)$. The figures are symmetric with respect to the sign of $n-2 \Omega$.

mined through a reproduction of the energy spectrum and binding energy of $A=481 f_{7 / 2}$ nuclei.

The different extent to which the GXPF1 interaction compared to the CD-Bonn and CD-Bonn+3terms interactions reflects development of pairing correlations and collective rotational modes in the upper $f p$ domain may be the reason why their pure two-body part do not correlate strongly as, for example, CDBonn and CD-Bonn+3terms do. Namely, in the scalar case the pure two-body correlations are 0.90 (between CD-Bonn and CD-Bonn+3terms) and only 0.56 (CDBonn and GXPF1) and 0.53 (CD-Bonn+3terms and GXPF1). In the isospin-scalar case, the correlations vary slightly with the particle number and isospin and they are on average, 0.88 (between CD-Bonn and CDBonn+3terms), 0.40 (CD-Bonn and GXPF1), and 0.37 (CD-Bonn+3terms and GXPF1). In addition, one can compare the significant monopole influence of the three interactions, which is very similar for all when averaged over the isospin values. However, in the isospin-

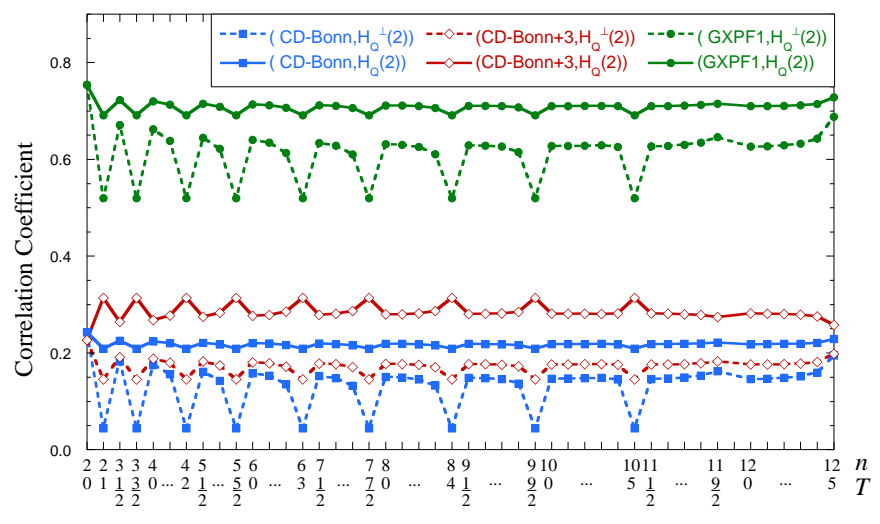

FIG. 2: Comparison between the orthogonal $H_{Q}^{\perp}(2)$ and the full two-body $H_{Q}(2)$ quadrupole-quadrupole interactions in their correlation to the realistic interactions, CDBonn (blue squares), CD-Bonn+3terms (red diamonds) and GXPF1 (green circles), in the isospin-scalar distribution. For each valence particle number, $n$, the isospin $T$ varies as $\frac{n}{2}, \frac{n}{2}-1, \ldots, 0(1)$. The figure is symmetric with respect to the sign of $n-2 \Omega$.

scalar distribution, the correlation coefficients involving the induced effective one-body contribution differ between GXPF1 and the two CD-Bonn interactions (Fig. 3). Their behavior, especially below mid-shell, reflects the similarity of the corresponding $T=0$ induced singleparticle energies (Appendix Eq. 17) and the opposite signs of the corresponding $\lambda_{3 / 2}^{T=1}$ (for $2 p_{3 / 2}$ ) and $\lambda_{5 / 2}^{T=1}$ (for $1 f_{5 / 2}$ ) pure one-body interactions.

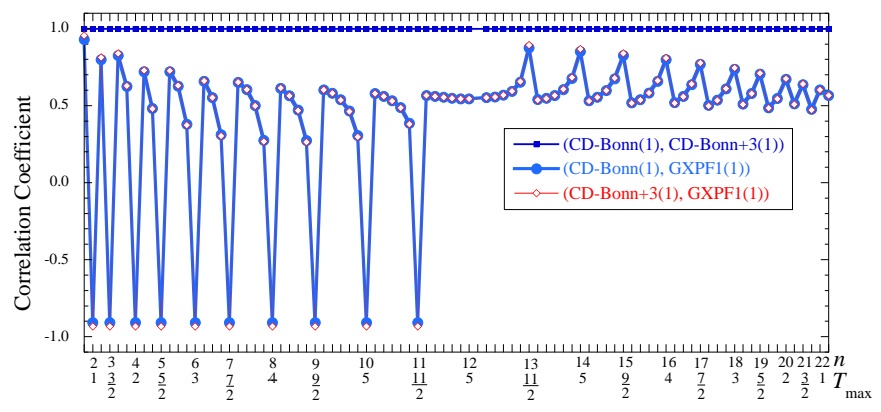

FIG. 3: Correlation coefficients between the pure one-body part of the CD-Bonn ,CD-Bonn+3terms and GXPF1 realistic interactions in the isospin-scalar distribution. For each valence particle number, $n$, the isospin $T$ varies as $T_{\max }, T_{\max }-1, \ldots, 0(1)$ with $T_{\max }=\min \left(\frac{n}{2}, \frac{4 \Omega-n}{2}\right)$.

In relation to the monopole (pure one-body) part of the realistic interactions and its significance in nuclear matter, an interesting outcome follows when each $H_{R}$ realistic interaction is compared to a model interaction [of the type (Eq. 3) or (Eq. 6)], in which the pure one-body part is provided by $H_{R}$ (excluding external s.p.en. for GXPF1) [14]. Such an interaction will be denoted by '*', e.g., $H_{M}^{*}=H_{M}(0)+H_{R}(1)+H_{M}(2)$. Namely, in the 
(isospin-)scalar case the $\zeta_{H_{R}, H_{M}^{*}}^{n(T)}$ correlation coefficient is on average 0.81 (0.84) for CD-Bonn, 0.79 (0.81) for CDBonn+3terms, and 0.84 (0.80) for GXPF1 (Fig. 4). This implies that pairing and quadrupole-quadrupole interactions can provide for a quite reasonable substitution of the two-body part of the interactions. Other types of two-body interactions different from pairing and $Q \cdot Q$ in both CD-Bonn interactions decrease quickly towards the mid-shell and beyond (e.g., down to only $4 \%$ of the total realistic interaction for the $T=n / 2$ states), where the role of the one-body part for shaping nuclear energy spectra strongly increases in importance.

\section{A. Individual-Orbit Analysis}

One can further perform a partitioning of the $f p$-space to single- $j$ orbits, $1 f_{7 / 2}, 1 f_{5 / 2}, 2 p_{1 / 2}$ and $2 p_{3 / 2}$, to provide for more detailed spectral measures that may reflect important fine effects that are otherwise averaged out when the entire $f p$ major shell is taken into account. We have already illustrated such an example and its significance by exploration of the $1 f_{7 / 2}$ orbit [12] (a few results from that study are presented here for completeness). Individual orbit analyses render correlation coefficients that are free of the influence of any one-body interaction [by definition, (Eq. 8,11,17)]. However, due to the small model space, the $2 p_{1 / 2}$ and $2 p_{3 / 2}$ orbits are combined in the analysis and hence $\varepsilon_{j}$ and $\lambda_{j}, j=\{1 / 2,3 / 2\}$, may be nonzero. This is why in the $2 p_{1 / 2} 2 p_{3 / 2}$ joint region we consider again only the two-body part of the realistic interactions and calculate correlation coefficients.

In the scalar distribution, a good portion, $53 \%$ to $98 \%$, of the extended pairing+quadrupole model interaction is described solely by the $H_{\mathfrak{s p}(4)}$ pairing interaction [Table II, $\left.\left(\zeta_{H_{M}, H_{\mathfrak{s p}(4)}}\right)^{2}\right]$. As mentioned above, although the $\mathrm{Sp}(4)$ breaking due to the quadrupole-quadrupole interaction $\left[H_{Q}^{\perp}(2)\right]$ is not large, correlations of $H_{R}$ with the full $H_{Q}(2)$ are sometimes even stronger than $\zeta_{H_{R}, H_{\mathfrak{s p}(4)}}$. The realistic interactions exhibit a quite well-developed rotational character (Table II, $\zeta_{H_{R}, H_{Q}(2)}$ ) except for CDBonn and CD-Bonn+3terms in the $2 p_{1 / 2} 2 p_{3 / 2}$ region. Besides these cases, the $H_{M}$ model interaction, as revealed by $\zeta_{H_{R}, H_{M}}$ in Table II, can be used as a very good approximation to the realistic interactions within each of the domains considered.

The extent to which the $\operatorname{Sp}(4)$ isovector $(T=1)$ pairing governs the interaction between two nucleons $(n=2)$ within a certain domain of states is represented by the correlation coefficients between $H_{\mathfrak{s p}(4)}$ and $H_{R}$ in the $T=1$ region (Table III). The results show large $J=0$ isovector coherence within each single- $j$ shell, $\zeta_{H_{\mathfrak{s p}(4)}^{T}, H_{R}}$, particularly for CD-Bonn+3terms and GXPF1, which are expected to describe quite well phenomena of a pairing character, while for CD-Bonn other types of interaction compete with pair formation. The latter are of the $H_{Q}^{\perp}(2)$ type for the $1 f_{7 / 2}$ and $1 f_{5 / 2}$ orbits, where
TABLE II: Correlation coefficients for many-nucleon systems of the $H_{R}$ realistic interactions with $H_{\mathfrak{s p}(4)}(\mathrm{Eq} .3)$, with the extended pairing+quadrupole model interaction $H_{M}$ (Eq. 6), and with the pure two-body full quadrupole-quadrupole interaction, $H_{Q}(2)$. The $\left(\zeta_{H_{M}, H_{\mathfrak{s} p(4)}}\right)^{2}$ quantity gives the part, in \%, of $H_{M}$ that is $\operatorname{Sp}(4)$ symmetric.

\begin{tabular}{|c|c|c|c|c|}
\hline \multicolumn{5}{|c|}{ Scalar Distribution } \\
\hline & & $1 f_{7 / 2}$ & $1 f_{5 / 2}$ & $2 p_{1 / 2} 2 p_{3 / 2}{ }^{a}$ \\
\hline \multirow[t]{4}{*}{ CD-Bonn } & $\zeta_{H_{R}, H_{\mathfrak{s p}(4)}}$ & 0.66 & 0.70 & 0.58 \\
\hline & $\zeta_{H_{R}, H_{M}}$ & 0.81 & 0.76 & 0.61 \\
\hline & $\left(\zeta_{H_{M}, H_{\mathfrak{s p}(4)}}\right)^{2}$ & $65.9 \%$ & $83.7 \%$ & $91.7 \%$ \\
\hline & $\zeta_{H_{R}, H_{Q}(2)}$ & 0.69 & 0.64 & 0.17 \\
\hline CD-Bonn & $\zeta_{H_{R}, H_{\mathfrak{s p}(4)}}$ & 0.64 & 0.67 & 0.51 \\
\hline \multirow[t]{3}{*}{+3 terms } & $\zeta_{H_{R}, H_{M}}$ & 0.87 & 0.86 & 0.52 \\
\hline & $\left(\zeta_{H_{M}, H_{\mathfrak{s p}(4)}}\right)^{2}$ & $53.4 \%$ & $60.6 \%$ & $98.3 \%$ \\
\hline & $\zeta_{H_{R}, H_{Q}(2)}$ & 0.80 & 0.82 & 0.22 \\
\hline \multirow[t]{4}{*}{ GXPF1 } & $\zeta_{H_{R}, H_{\mathfrak{s p}(4)}}$ & 0.76 & 0.77 & 0.70 \\
\hline & $\zeta_{H_{R}, H_{M}}$ & 0.93 & 0.84 & 0.90 \\
\hline & $\left(\zeta_{H_{M}, H_{\mathfrak{s p}(4)}}\right)^{2}$ & $67.9 \%$ & $84.7 \%$ & $61.7 \%$ \\
\hline & $\zeta_{H_{R}, H_{Q}(2)}$ & 0.78 & 0.69 & 0.85 \\
\hline
\end{tabular}

${ }^{a}$ pure two-body part of the interactions

the residual interactions are negligible. In short, the simple $\mathrm{Sp}(4)$ model interaction and especially its extended pairing+quadrupole $H_{M}$ interaction can reproduce reasonably well the $T=1$ part of the realistic interactions under consideration in the orbits specified in Table III.

TABLE III: Correlation coefficients for a two-nucleon system $(n=2)$ of the $H_{R}$ realistic interactions with $H_{\mathfrak{s p}(4)}(\mathrm{Eq} .3)$ and with the extended pairing+quadrupole model interaction $H_{M}$ (Eq. 6). The $\left(\zeta_{H_{M}, H_{\mathfrak{s p}(4)}}\right)^{2}$ quantity gives the part, in $\%$, of $H_{M}$ that is $\operatorname{Sp}(4)$ symmetric.

\begin{tabular}{|c|c|c|c|c|}
\hline \multicolumn{5}{|c|}{ Isospin-scalar Distribution, $T=1$} \\
\hline & & $1 f_{7 / 2}$ & $1 f_{5 / 2}$ & $2 p_{1 / 2} 2 p_{3 / 2}{ }^{a}$ \\
\hline \multirow[t]{3}{*}{ CD-Bonn } & $\zeta_{H_{R}, H_{\mathfrak{s p}(4)}^{T}}^{T=1}$ & 0.61 & 0.57 & 0.54 \\
\hline & $\zeta_{H_{R}, H_{M}}^{T=1}$ & 0.95 & 1.00 & 0.59 \\
\hline & $\left(\zeta_{H_{M}, H_{\mathfrak{s p}(4)}}\right)^{2}$ & $41.5 \%$ & $32.3 \%$ & $84.2 \%$ \\
\hline CD-Bonn & $\zeta_{H_{R}, H_{\mathfrak{s p}(4)}}^{T=1}$ & 0.85 & 0.93 & 0.59 \\
\hline \multirow[t]{2}{*}{+3 terms } & $\zeta_{H_{R}, H_{M}}^{T=1}$ & 0.98 & 1.00 & 0.61 \\
\hline & $\left(\zeta_{H_{M}, H_{\mathfrak{s p}(4)}}\right)^{2}$ & $73.9 \%$ & $86.9 \%$ & $92.1 \%$ \\
\hline \multirow[t]{3}{*}{ GXPF1 } & $\zeta_{H_{R}, H_{\mathfrak{s p}(4)}^{T}}^{T=1}$ & 0.71 & 0.86 & 0.69 \\
\hline & $\zeta_{H_{R}, H_{M}}^{T=1}$ & 0.96 & 1.00 & 0.94 \\
\hline & $\left(\zeta_{H_{M}, H_{\mathfrak{s p}(4)}}\right)^{2}$ & $54.5 \%$ & $74.0 \%$ & $53.5 \%$ \\
\hline
\end{tabular}

${ }^{a}$ pure two-body part of the interactions

For nuclear systems with more than two nucleons and states with $T=n / 2$, Table III continues to apply. In the $T \neq n / 2$ cases, the realistic interactions have a very sim- 


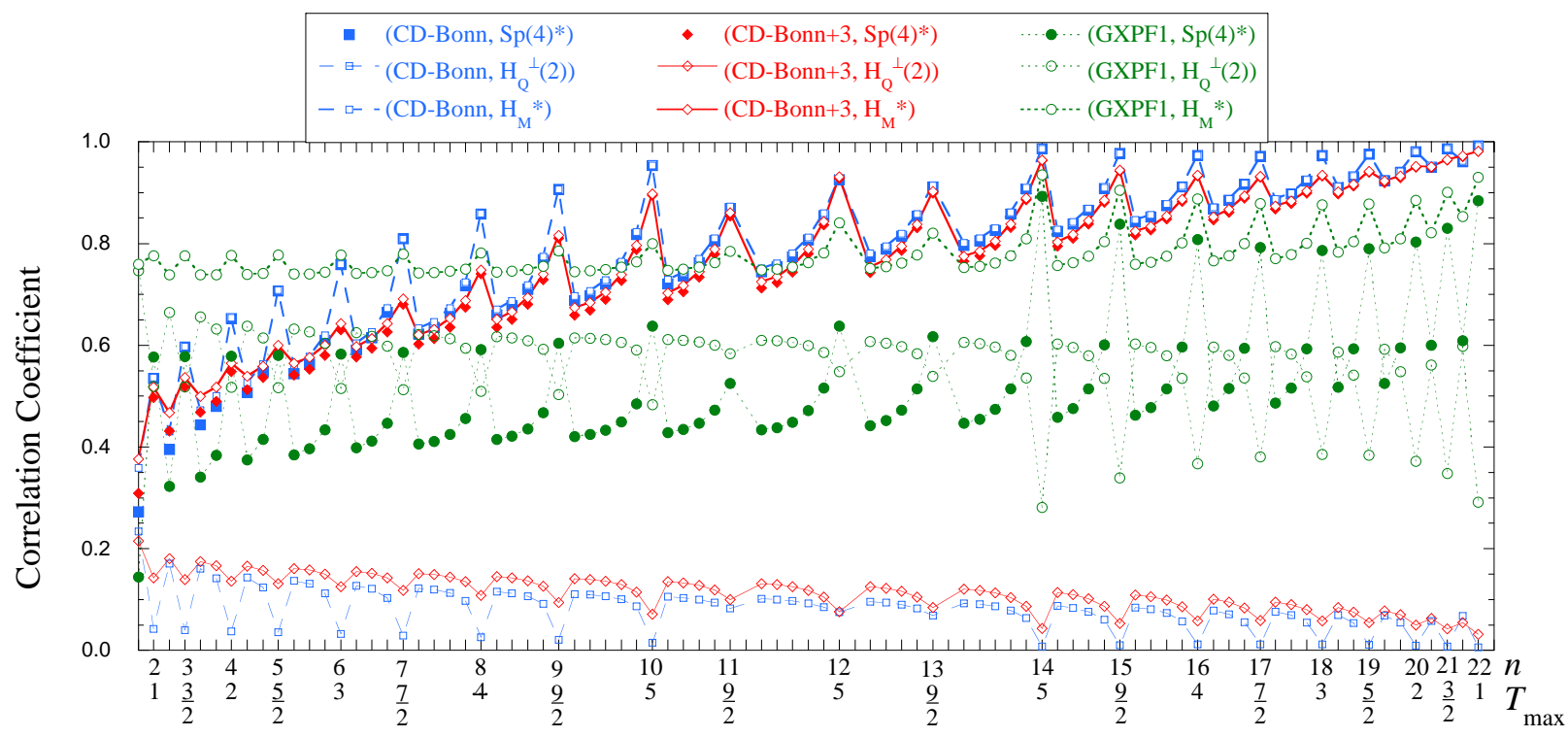

FIG. 4: Correlation coefficients in the isospin-scalar distribution of the realistic interactions, CD-Bonn (blue squares), CDBonn+3terms (red diamonds) and GXPF1 (green circles), with $H_{\mathfrak{s p}(4)}^{*}$ (filled symbols), $H_{Q}^{\perp}(2)$ (transparent symbols) and $H_{M}^{*}$ (empty symbols), which closely follows $\zeta_{H_{R}, H_{\mathfrak{s p}(4)}^{*}}$ for both CD-Bonn interactions, in the upper $f p$ shell. For each valence particle number, $n$, the isospin $T$ varies as $T_{\max }, T_{\max }-1, \ldots, 0(1)$ with $T_{\max }=\min \left(\frac{n}{2}, \frac{4 \Omega-n}{2}\right)$. A complete explanation of the notation is given in the text.

ilar behavior in both the $1 f_{5 / 2}$ and $1 f_{7 / 2}$ (refer to Fig. 4 in [12] for correlations of $H_{R}$ to the extended pairing+quadrupole model interaction) where CD-Bonn is dominated by $H_{Q}^{\perp}(2)$, while both $H_{Q}^{\perp}(2)$ and $H_{\mathfrak{s p}(4)}$ contributions enter at almost the same footing in CDBonn+3terms. In the $2 p_{1 / 2} 2 p_{3 / 2}$ joint region the main contribution to GXPF1 comes from $H_{\mathfrak{s p}(4)}$ for all $n, T$ values. The GXPF1 interaction in the $1 f_{5 / 2} 1 f_{7 / 2}$ orbits and both CD-Bonn interactions in the $2 p_{1 / 2} 2 p_{3 / 2}$ retain the same overall pattern as in the upper $f p$ region (Fig. 1) and with relatively stronger correlations coefficients.

A very close similarity is observed between realistic and model interactions within individual orbits. However, more prominent differences among the interactions appear in the multi- $j$ upper $f p$ domain especially concerning both CD-Bonn interactions. This may indicate that the inter-orbit interactions do not respect strongly the symmetries imposed in the model interactions. In addition, the interaction strengths may differ from one orbit to another. While they do not affect correlation coefficients in the singe- $j$ cases, their relative strength is of a great importance for multi- $j$ analysis.

\section{B. Energy Spectra for $A=58$ Nuclei}

The results presented above show that the $H_{M}$ model Hamiltonian (Eq. 6) can be used in the upper $f p$ region as a quite good approximation of the pure two-body part of the GXPF1 interaction (Fig. 1). The model interaction can also provide for a reasonable substitu- tion of the pure two-body part of CD-Bonn and CDBonn+3terms, especially towards and beyond the midshell and for $T \approx n / 2$ states in the isospin-scalar case (Fig. 4). As an illustrative example, we apply the simpler model interaction (Eq. 6) to a nuclear system of two nucleons in the upper $f p$ region without any parameter variation.

Particularly, we assume a ${ }^{56} \mathrm{Ni}$-core and that both nucleons in ${ }^{58} \mathrm{Ni}$ and ${ }^{58} \mathrm{Cu}$ occupy the upper $f p$ orbits with reasonable probability [11]. For a description of the lowlying structure of these nuclei, external single-particle energies are needed to rescale at the end the eigenvalues of the model Hamiltonian. This is performed trivially due to the microscopic structure of the model eigenstates, which are constructed in terms of fermion creation operators. We adopt single-particle energies that are derived from the ${ }^{57} \mathrm{Ni}$ energy spectrum. To a very reasonable degree, these energies reflect the influence of the $1 f_{7 / 2}$ orbit and the core mean-field contribution.

The model Hamiltonian uses a $\chi$ value of 0.027 that we obtained through a comparison of $H_{M}$ to GXPF1 within a scalar distribution. The reason is that the pure twobody GXPF1 interaction holds the best correlation coefficient to the model interaction in the upper $f p$ region (Table I). In addition, the energy spectra for ${ }^{58} \mathrm{Ni}$ and ${ }^{58} \mathrm{Cu}$ are found to be closely reproduced by shell model calculations with the GXPF1 interaction in the full $f p$ oscillator shell [11].

We extend the model space to include the $1 g_{9 / 2}$ orbit as it intrudes in the upper $f p$ domain. This is exactly the space where the $\mathrm{Sp}(4)$ model was applied and interaction 
strength parameters determined [21]. The latter are kept fixed throughout the present analyses. The results (Fig. 5 ) show a very good reproduction of the low-lying $T=1$ spectra in ${ }^{58} \mathrm{Ni}$ and ${ }^{58} \mathrm{Cu}$, especially the lowest $2^{+}$states for both nuclei and the first $0^{+}(T=1)$ state above the ${ }^{58} \mathrm{Cu}$ ground state. Both states are of particular significance. On the one hand, the energy difference between the lowest $0^{+}$and $2^{+}$states is believed to be directly affected by the formation of correlated pairs in the lowest $0^{+}$state (ground state for even-even nuclei) and the pairing gap that occurs below the first excited $2^{+}$state of a broken pair. On the other hand, the $0^{+}(T=1)$ to $1^{+}(T=0)$ energy difference in $N=Z$ odd-odd nuclei is associated with the close interplay of isovector $(T=1$, pairing correlations) and isoscalar $(\mathrm{T}=0)$ interactions between protons and neutrons in the same major shell. In addition, the $T=0$ spectrum of ${ }^{58} \mathrm{Cu}$ as predicted by the model interaction possesses the same pattern of the levels observed, namely, the $1^{+}$ground state is followed by $3^{+}, 1^{+}, 2^{+}, 4^{+}$, and $3^{+}$. The ${ }^{58} \mathrm{Cu} T=0$ spectrum appears narrower than the experimental data, which suggests that different interaction strengths for $T=0$ and $T=1$ need to be used.

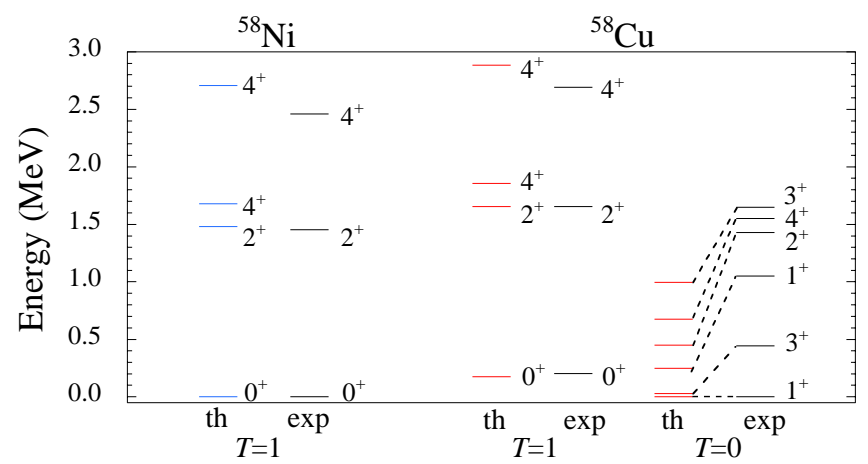

FIG. 5: Theoretical ('th') low-lying energy spectra for ${ }^{58} \mathrm{Ni}$ (left, blue) and ${ }^{58} \mathrm{Cu}$ (right, red) compared to experiment ('exp', black). The theoretical calculations are performed in the $1 f_{5 / 2} 2 p_{1 / 2} 2 p_{3 / 2} 1 g_{9 / 2}$ major shell with the $H_{M}$ model interaction (Eq. 6 with $\chi=0.027$ ) and with single-particle energies derived from ${ }^{57} \mathrm{Ni}$ experimental energy levels.

In short, we demonstrate that strong correlations typically yield very similar energy spectra and reproduction of the overall pattern of the energy levels without any adjustment of the interaction strength parameters.

\section{CONCLUSIONS}

We compared three modern realistic interactions, CDBonn, CD-Bonn+3terms, and GXPF1 and two model pairing and quadrupole interactions in the upper $f p$ region by means of the theory of spectral distributions. We focus on the weaker but property-defining two-body part of the interactions, which yields strong correlation of the pure two-body GXPF1 realistic interaction with the pairing+quadrupole extended model in both scalar and isospin-scalar distributions in the upper $f p$ domain.

Particularly, the outcomes show very good scalardistribution correlations of GXPF1 with the $\mathrm{Sp}(4)$ dynamically symmetric interaction, which includes isovector pairing correlations and a proton-neutron isoscalar force, and with the quadrupole-quadrupole interaction for description of SU(3) dynamically symmetric collective rotational modes. Rotational features within manynucleon systems in the upper $f p$ domain are found to be more fully developed for GXPF1 and less for CDBonn+3terms and CD-Bonn. The isospin-scalar space partitioning demonstrates a tendency in GXPF1 towards the formation of correlated pairs in the highest possible isospin groups of states. For both CD-Bonn interactions, other types of two-body interactions different from the pairing and quadrupole interactions (with fixed strength throughout the upper $f p$ shell) decrease in importance towards and beyond the mid-shell until they become negligible, that is, only about $4 \%$ of the total realistic interaction.

Individual orbit analysis, including the $1 f_{7 / 2}, 1 f_{5 / 2}$, $2 p_{1 / 2}$, and $2 p_{3 / 2}$ levels, shows considerably stronger correlation of all the interactions with the pairing+quadrupole model interaction (up to $0.8-1.00$ ). In addition, the results, independent of pairing and quadrupole strengths, reveal a tendency for pair formation within the individual orbits for the $T=1$ part of the CD-Bonn+3terms and GXPF1 interactions and well developed collective rotational features within the $1 f_{7 / 2}$ and $1 f_{5 / 2}$ orbits especially in CD-Bonn +3 terms and the $T=1$ CD-Bonn interactions.

In the upper $f p$ region, the extended $H_{M}$ pairing+quadrupole interaction is strongly correlated with the pure two-body GXPF1 realistic interaction especially in the scalar distribution and for this reason can be used as a good approximation. This is reflected in the quite good agreement between the experimental lowlying energy spectra of ${ }^{58} \mathrm{Ni}$ and ${ }^{58} \mathrm{Cu}$ and the theoretical prediction based on the $H_{M}$ model interaction in the $1 f_{5 / 2} 2 p_{1 / 2} 2 p_{3 / 2} 1 g_{9 / 2} \quad$ major shell.

In summary, based on these results, spectral distribution theory appears to be a good framework for uncovering fundamental properties of realistic interactions in many-nucleon systems. We find varying degrees of respect for selected underlying symmetries. As some of these symmetries have been demonstrated to be important for certain spectral features, we have a tool for rapidly assessing the likely success of these interactions for reproducing those spectral features. For example, it is unlikely that the CD-Bonn+3terms interaction will provide a fully satisfactory description of the rotational properties of nuclei in the upper $f p$ shell. Given that this interaction was determined only with $A=48 \mathrm{nu}-$ clear spectra and binding energies [10], future efforts at expanding the region of its validity in the no-core shell model should benefit from the analysis provided here. 


\section{Acknowledgments}

One of us (JV) would like to thank Sorina Popescu, Sabin Stoica and Geanina Negoita for valuable discussions. This work was supported by the US National Science Foundation, Grant Numbers 0140300 \& 0500291 , and the Southeastern Universities Research Association. This work was partly performed under the auspices of the US Department of Energy by the University of California, Lawrence Livermore National Laboratory under contract No. W-7405-Eng-48 and under the auspices of grants DE-FG02-87ER40371 \& DE-AC02-76SF00515.

\section{Appendix}

The theory of spectral distributions (or statistical spectroscopy) is well documented in the literature [3-5, 7, 41] and is accompanied by computational codes $[7,56]$ for evaluating various measures. The purpose of this appendix is to specify the notation and ensure that our definitions of the summations and numerical factors that enter into such measures are clearly understood.

In standard second quantized form, a one- and twobody interaction Hamiltonian is given in terms of fermion creation $a_{j m(1 / 2) \sigma}^{\dagger}=c_{j m(1 / 2) \sigma}^{\dagger}$ and annihilation $a_{j m(1 / 2) \sigma}=(-1)^{j+m+1 / 2+\sigma} c_{j-m(1 / 2)-\sigma}$ tensors, which create or annihilate a particle of type $\sigma= \pm 1 / 2$ (proton/neutron) in a state of total angular momentum $j$ (half integer) with projection $m$ in a finite space $2 \Omega=$ $\Sigma_{j}(2 j+1)$,

$$
\begin{gathered}
H=-\sum_{r \leq s} \sqrt{[r]} \varepsilon_{r s}\left\{a_{r}^{\dagger} \otimes a_{s}\right\}^{(00)}-\sum_{\substack{r \leq s \\
t \leq u \\
\Gamma=(J, T)}} \frac{\sqrt{[\Gamma]}}{\sqrt{\left(1+\delta_{r s}\right)\left(1+\delta_{t u}\right)}} W_{r s t u}^{\Gamma}\left\{\left\{a_{r}^{\dagger} \otimes a_{s}^{\dagger}\right\}^{\Gamma} \otimes\left\{a_{t} \otimes a_{u}\right\}^{\Gamma}\right\}^{(00)} \\
=-\sum_{r \leq s} \sqrt{[r]} \varepsilon_{r s}\left\{a_{r}^{\dagger} \otimes a_{s}\right\}^{(00)}-\frac{1}{4} \sum_{\substack{r s t u \\
\Gamma}} \sqrt{\left(1+\delta_{r s}\right)\left(1+\delta_{t u}\right)[\Gamma]} W_{r s t u}^{\Gamma}\left\{\left\{a_{r}^{\dagger} \otimes a_{s}^{\dagger}\right\}^{\Gamma} \otimes\left\{a_{t} \otimes a_{u}\right\}^{\Gamma}\right\}^{(00)}, \\
\Gamma
\end{gathered}
$$

where the labels are $r=\left\{j_{r}, \tau_{r}=\frac{1}{2}\right\},[r]=2\left(2 j_{r}+1\right)$, and $[\Gamma]=(2 J+1)(2 T+1)$. In (Eq. 7), $\varepsilon_{r s}$ is the (external) single-particle energy (hereafter we consider no angular momentum degeneracy for two different radial quantum numbers, $\varepsilon_{r s}=\varepsilon_{r} \delta_{r s}$ ) and $W_{r s t u}^{J T}$ is the two-body antisymmetric matrix element in the JT-coupled scheme $\left[W_{r s t u}^{\Gamma}=-(-)^{r+s-\Gamma} W_{s r t u}^{\Gamma}=\right.$ $\left.-(-)^{t+u-\Gamma} W_{r s u t}^{\Gamma}=(-)^{r+s-t-u} W_{\text {srut }}^{\Gamma}=W_{\text {turs }}^{\Gamma}\right]$. For an isospin nonconserving two-body interaction of isospin rank $\mathcal{T}$, the coupling of fermion operators is as follows, $\left\{\left\{a_{r}^{\dagger} \otimes a_{s}^{\dagger}\right\}^{J T} \otimes\left\{a_{t} \otimes a_{u}\right\}^{J T}\right\}^{(0 \mathcal{T})}$, with $W_{\text {rstu }}^{(\mathcal{T}) J T}$ matrix elements.

For a major shell that consists of several $s$ orbits, each of degeneracy $\mathcal{N}_{s}\left(\mathcal{N}=\sum_{s} \mathcal{N}_{s}\right)$, the (traceless) external single-particle energy of the $r^{\text {th }}$ orbit is obtained as

$$
\tilde{\varepsilon}_{r}=\varepsilon_{r}-\varepsilon=\varepsilon_{r}-\frac{1}{\mathcal{N}} \sum_{s} \varepsilon_{s} \mathcal{N}_{s},
$$

where the average (external) single-particle energy is

$$
\varepsilon=\frac{1}{\mathcal{N}} \sum_{s} \varepsilon_{s} \mathcal{N}_{s}
$$

Scalar Distribution. For a two-particle system, the monopole moment (centroid), which is the average expectation value of the two-body interaction, is defined in the scalar case as

$$
\begin{aligned}
W_{c} & =\frac{1}{\left(\begin{array}{c}
\mathcal{N} \\
2
\end{array}\right)} \sum_{r \leq s, \Gamma}[\Gamma] W_{r s r s}^{\Gamma} \\
& =\frac{\sum_{r s, \Gamma}[\Gamma] W_{r s r s}^{\Gamma}\left(1+\delta_{r s}\right)}{\mathcal{N}(\mathcal{N}-1)},
\end{aligned}
$$

where $\mathcal{N}=4 \Omega=2 \sum_{r}\left(2 j_{r}+1\right)$, the $\Gamma$-sum goes over all possible $(J, T)$ for given $r, s$, and $\left(\begin{array}{c}\mathcal{N} \\ 2\end{array}\right)=\sum_{r \leq s, \Gamma}[\Gamma]$. The traceless induced single-particle energy is constructed by contraction of the two-body interaction into an effective one-body operator under the particular group structure,

$$
\begin{aligned}
\lambda_{r}= & \frac{1}{\mathcal{N}_{r}} \sum_{s, J T}[J T] W_{r s r s}^{J T}\left(1+\delta_{r s}\right) \\
& -\frac{1}{\mathcal{N}} \sum_{t u, J T}[J T] W_{t u t u}^{J T}\left(1+\delta_{t u}\right) .
\end{aligned}
$$

For a system with one hole in the $r^{\text {th }}$ orbit, $\lambda_{r}$ corresponds to the energy of a single particle as contributed by the interaction with the valence particles above the 
core and differs from the $\epsilon_{r}$, which are generated by the interaction of a single particle with the core. In turn, the traceless pure two-body interaction is defined as

$$
W_{r s t u}^{J T}(2)=W_{r s t u}^{J T}-\left(W_{c}+\frac{\lambda_{r}+\lambda_{s}}{\mathcal{N}-2}\right) \delta_{r t} \delta_{s u} .
$$

For the $\mathrm{Sp}(4)$ interaction, the average two-body interaction is expressed in terms of the parameters in the following way,

$$
W_{c}=-\frac{3 G_{0}}{\left(\begin{array}{c}
\mathcal{N} \\
2
\end{array}\right)}+\frac{3 E_{0}}{2(\mathcal{N}-1)}-C .
$$

The pure one-body (traceless) induced single-particle en- ergies are zero due to degeneracies in both cases of a single- $j$ orbit or the $1 f_{5 / 2} 2 p_{1 / 2} 2 p_{3 / 2} 1 g_{9 / 2}$ multi- $j$ shell. In addition, the pure two-body matrix elements are independent of the $C$ parameter and hence the correlation coefficient depends only on two model parameters, $G_{0}$ and $E_{0}$.

In order to calculate energy moments and their propagation for higher $n$ (and $T$ ) values, each interaction $H$ (consisting of one $(k=1)$ - and two $(k=2)$-body parts) needs to be expressed as a linear combination of terms of definite particle rank (irreducible tensors $\mathcal{H}_{k}(\nu)$ of rank $\nu=0,1,2)$, that is as a collection of pure zero-, one- and two-body interactions. For $n$ particles, the Hamiltonian can be rendered,

$$
\begin{aligned}
H= & n \mathcal{H}_{1}(0)+\left(\begin{array}{l}
n \\
2
\end{array}\right) \mathcal{H}_{2}(0)+\mathcal{H}_{1}(1)+(n-1) \mathcal{H}_{2}(1)+\mathcal{H}_{2}(2) \\
= & -n \varepsilon-\left(\begin{array}{c}
n \\
2
\end{array}\right) W_{c}-\sum_{r}[r]^{\frac{1}{2}}\left(\tilde{\varepsilon}_{r}+\frac{n-1}{\mathcal{N}-2} \lambda_{r}\right)\left\{a_{r}^{\dagger} \otimes a_{r}\right\}^{(00)} \\
& -\sum_{\substack{r \leq s \\
t \leq u \\
\Gamma=(J, T)}} \frac{\sqrt{[\Gamma]}}{\sqrt{\left(1+\delta_{r s}\right)\left(1+\delta_{t u}\right)}} W_{r s t u}^{\Gamma}(2)\left\{\left\{a_{r}^{\dagger} \otimes a_{s}^{\dagger}\right\}^{\Gamma} \otimes\left\{a_{t} \otimes a_{u}\right\}^{\Gamma}\right\}^{(00)}
\end{aligned}
$$

for then the quantity that defines the correlation coefficient (Eq. 1) is easily computed for different particle numbers $n$,

$$
\begin{aligned}
\left\langle H^{\dagger} H^{\prime}\right\rangle^{n}-\left\langle H^{\dagger}\right\rangle^{n}\left\langle H^{\prime}\right\rangle^{n}= & \frac{n(\mathcal{N}-n)}{\mathcal{N}(\mathcal{N}-1)} \sum_{r}\left[\tilde{\varepsilon}_{r}+\frac{n-1}{\mathcal{N}-2} \lambda_{r}\right]\left[\tilde{\varepsilon}_{r}^{\prime}+\frac{n-1}{\mathcal{N}-2} \lambda_{r}^{\prime}\right] \mathcal{N}_{r} \\
& +\frac{n(n-1)(\mathcal{N}-n)(\mathcal{N}-n-1)}{\mathcal{N}(\mathcal{N}-1)(\mathcal{N}-2)(\mathcal{N}-3)} \sum_{\substack{r \leq s \\
t \leq u \\
\Gamma}}[\Gamma] W_{r s t u}^{\Gamma}(2) W_{r s t u}^{\prime \Gamma}(2) . \\
\Gamma=(J, T) &
\end{aligned}
$$

Isospin-Scalar Distribution. Analogously, the centroid in the isospin-scalar case is defined as,

$$
W_{c}^{T}=\frac{2}{\mathcal{N}\left(\mathcal{N}+(-1)^{T}\right)} \sum_{r \leq s, J}[J] W_{r s r s}^{J T}
$$

where $\mathcal{N}=2 \Omega$. The $\lambda_{r}^{T}$ traceless induced single-particle energy for orbit $r$ and the $W_{r s t u}^{J T}(2)$ traceless pure twobody interaction [7] are defined as,

$$
\begin{aligned}
\lambda_{r}^{T} & =\frac{1}{\mathcal{N}_{r}} \sum_{s, J}[J] W_{\text {rsrs }}^{J T}\left(1+\delta_{r s}\right) \\
& -\frac{1}{\mathcal{N}} \sum_{t u, J}[J] W_{t u t u}^{J T}\left(1+\delta_{t u}\right),
\end{aligned}
$$

In order to calculate the correlation coefficient $\zeta^{n, T}$ and the variance $\sigma^{n, T}$, the following quantities are needed, 


$$
\begin{aligned}
&\left\langle H^{\dagger} H^{\prime}\right\rangle^{n, T}-\left\langle H^{\dagger}\right\rangle^{n, T}\left\langle H^{\prime}\right\rangle^{n, T}= p_{1}(T) \sum_{r} \mathcal{N}_{r} \tilde{\varepsilon}_{r} \tilde{\varepsilon}_{r}^{\prime}+\sum_{r, \tau} p_{1}(n, T, \tau) \mathcal{N}_{r}\left[\tilde{\varepsilon}_{r} \lambda_{r}^{\prime \tau}+\tilde{\varepsilon}_{r}^{\prime} \lambda_{r}^{\tau}\right] \\
&+\sum_{r,\left\{\tau_{1}, \tau_{2}\right\}} p_{1}\left(n, T, \tau_{1}, \tau_{2}\right) \mathcal{N}_{r}\left[\lambda_{r}^{\tau_{1}} \lambda_{r}^{\prime \tau_{2}}+\lambda_{r}^{\tau_{2}} \lambda_{r}^{\prime \tau_{1}}\right] \\
&+\sum_{\tau} p_{2}(n, T, \tau) \frac{2}{\mathcal{N}\left(\mathcal{N}+(-1)^{\tau}\right)} \sum_{\substack{r \leq s \\
t \leq u \\
J}}[J] W_{r s t u}^{J \tau}(2) W_{r s t u}^{\prime J \tau}(2), \\
& J
\end{aligned}
$$

where $\tau$ is 0 or 1 , and the sum over $\left\{\tau_{1}, \tau_{2}\right\}$ goes over the set of values, $\{0,0\},\{0,1\}$ and $\{1,1\}$. The propagator functions are derived in $[5,57]$ to be,

$$
\begin{aligned}
p_{1}(T)= & \frac{n(\mathcal{N}+2)\left(\mathcal{N}-\frac{n}{2}\right)-2 \mathcal{N} T(T+1)}{\mathcal{N}(\mathcal{N}-1)(\mathcal{N}+1)} \\
p_{1}(n, T, \tau)= & \frac{(\mathcal{N}+2)\left(\mathcal{N}-\frac{n}{2}\right)\left[(2 \tau+1) n\left(n+2(-1)^{\tau}\right)-4 T(T+1)(-1)^{\tau}\right]+4 \mathcal{N} T(T+1)(1-n)\left(1-(-1)^{\tau}\right)}{4 \mathcal{N}(\mathcal{N}-1)(\mathcal{N}+1)\left(\mathcal{N}+2(-1)^{\tau}\right)}(21) \\
p_{1}\left(n, T, \tau_{1}, \tau_{2}\right)= & \frac{1}{8(\mathcal{N}-1)(\mathcal{N}+1)(\mathcal{N}-2)\left(\mathcal{N}+2(-1)^{\tau_{1}}\right)}\left\{4 \mathcal{N} T(T+1)(n-1)(\mathcal{N}-2 n+4)\left(1-(-1)^{\tau_{1}}\right)\right. \\
& +\left[\left(2 \tau_{1}+1\right) n\left(n+2(-1)^{\tau_{1}}\right)-4 T(T+1)(-1)^{\tau_{1}}\right]\left[\left(2 \tau_{2}+1\right)\left(n+2(-1)^{\tau_{2}}\right)\left(\mathcal{N}-\frac{n}{2}\right) \frac{1}{2}+T(T+1)(-1)^{\tau_{2}}\right] \\
& \left.\times\left[\mathcal{N}-2(-1)^{\tau_{2}}\right]\right\} \\
p_{2}(n, T, \tau=0)= & \frac{[n(n+2)-4 T(T+1)]\left[\left(\mathcal{N}-\frac{n}{2}\right)\left(\mathcal{N}-\frac{n}{2}+1\right)-T(T+1)\right]}{8 \mathcal{N}(\mathcal{N}-1)} \\
p_{2}(n, T, \tau=1)= & \frac{1}{\mathcal{N}(\mathcal{N}+1)(\mathcal{N}-2)(\mathcal{N}-3)}\left\{\frac{1}{2} T^{2}(T+1)^{2}(3 \mathcal{N}-7 \mathcal{N}+6)\right. \\
& +\frac{3}{8} n(n-2)\left(\mathcal{N}-\frac{n}{2}\right)\left(\mathcal{N}-\frac{n}{2}+1\right)(\mathcal{N}+1)(\mathcal{N}+2) \\
& \left.+\frac{1}{2} T(T+1)\left[(5 \mathcal{N}-3)(\mathcal{N}+2) n\left(\frac{n}{2}-\mathcal{N}\right)+\mathcal{N}(\mathcal{N}-1)(\mathcal{N}+1)(\mathcal{N}+6)\right]\right\} .
\end{aligned}
$$

For the $\operatorname{Sp}(4)$ interaction, the centroid energy for a given isospin value is,

$$
W_{c}^{T}=-\frac{G_{0}}{\left(\begin{array}{c}
\mathcal{N} \\
2
\end{array}\right)} \delta_{T 1}+E_{0}\left[(-1)^{T}+\frac{1}{2}\right]-C .
$$

The pure two-body matrix elements, $W_{r s t u}^{J T}(2)$, and hence the correlation coefficients involving $H_{\mathfrak{s p}(4)}$, are then independent of the $C$ and $E_{0}$ parameters. The $\operatorname{Sp}(4)$ matrix elements (Eq. 4) do not contribute to the one-body part of the Hamiltonian, that is all $\lambda_{r}^{T}$ are zero for a single- $j$ orbit as well as for the $1 f_{5 / 2} 2 p_{1 / 2} 2 p_{3 / 2} 1 g_{9 / 2}$ major shell. In addition, the contribution of the external single-particle energies is already accounted for on average by the term $\varepsilon \hat{N}$ in (Eq. 3) and hence $\tilde{\varepsilon}_{r}=0$. Therefore, there is no pure one-body part in the $\operatorname{Sp}(4)$ model Hamiltonian. As far as the realistic interactions are concerned, $\tilde{\varepsilon}_{r}$ and $\lambda_{r}^{(T)}$ are trivially zero for the single$j$ case. For CD-Bonn and CD-Bonn+3terms within a multi- $j$ shell there are no explicit external single-particle energies, $\varepsilon_{r}=0$, and hence the one-body contribution emerges only from their $W_{r s t u}^{J T}$ matrix elements.
[1] R. Machleidt, F. Sammarruca, and Y. Song, Phys. Rev. C53, R1483 (1996); R. Machleidt, Phys. Rev. C63, 024001 (2001).

[2] D. R. Entem and R. Machleidt, Phys. Rev. C68, 041001 (2003).

[3] J. B. French and K. F. Ratcliff, Phys. Rev. C3, 94 (1971).

[4] F. S. Chang, J. B. French, and T. H. Thio, Ann. Phys. (N.Y.) 66, 137 (1971).
[5] K. T. Hecht and J. P. Draayer, Nucl. Phys. A223, 285 (1974).

[6] T. R. Halemane, K. Kar, and J. P. Draayer, Nucl. Phys. A311, 301 (1978).

[7] V. K. B. Kota, Phys. Rev. C20, 347 (1979); Fortran Programs for Statistical Spectroscopy Calculations.

[8] G. Rosensteel, Nucl. Phys. A341, 397 (1980).

[9] J. P. Draayer and G. Rosensteel, Nucl. Phys. A386, 189 
(1982).

[10] S. Popescu, S. Stoica, J. P. Vary, and P. Navratil, to be published.

[11] M. Honma, T. Otsuka, B. A. Brown, and T. Mizusaki, Phys. Rev. C69, 034335 (2004).

[12] K. D. Sviratcheva, J. P. Draayer, and J. P. Vary, Phys. Rev. C73, 034324 (2006).

[13] J. P. Draayer, Nucl. Phys. A216, 457 (1973).

[14] V. K. B. Kota, S. P. Pandya, and V. Potbhare, Nucl. Phys. A349, 397 (1980).

[15] C. R. Countee, J. P. Draayer, T. R. Halemane, and K. Kar, Nucl. Phys. A356, 1 (1981).

[16] J. P. Elliott, Proc. Roy. Soc. (London) A245, 128 (1958); A245, 562 (1958); J. P. Elliott and M. Harvey, Proc. Roy. Soc. (London) A272, 557 (1963).

[17] K. T. Hecht, Nucl. Phys. 63, 177 (1965); Phys. Rev. 139, B794 (1965); Nucl. Phys. A102, 11 (1967); J. N. Ginocchio, Nucl. Phys. 74, 321 (1965).

[18] J. Engel, K. Langanke, and P. Vogel, Phys. Lett. B389, 211 (1996).

[19] K. Kaneko, M. Hasegawa, and J. Zhang, Phys. Rev. C59, 740 (1999).

[20] K. D. Sviratcheva, A. I. Georgieva, and J. P. Draayer, J. Phys. G: Nucl. Part. Phys. 29, 1281 (2003).

[21] K. D. Sviratcheva, A. I. Georgieva, and J. P. Draayer, Phys. Rev. C70 064302 (2004).

[22] K. D. Sviratcheva, A. I. Georgieva, and J. P. Draayer, Phys. Rev. C69 024313 (2004).

[23] K. Langanke, Nucl. Phys. A630, 368c (1998).

[24] P. T. Hosmer et al., Phys. Rev. Lett. 94, 112501 (2005).

[25] E. Caurier, G. Martinez-Pinedo, F. Nowacki, A. Poves, and A. P. Zuker, Rev. Mod. Phys. 77, 427 (2005).

[26] B. A. Brown, Prog. Part. Nucl. Phys. 47, 517 (2001).

[27] T. Otsuka, M. Honma, T. Mizusaki, N. Shimizu, and Y. Utsuno, Prog. Part. Nucl. Phys. 47, 319 (2001).

[28] J. P. Draayer, J. B. French, V. Potbhare, and S. S. M. Wong, Phys. Lett. 55B, 263, 349 (1975); J. P. Draayer, J. B. French, M. Prasad, V. Potbhare, and S. S. M. Wong, Phys. Lett. 57B, 130 (1975); J. P. Draayer, J. B. French, and S. S. M. Wong, Ann. of Phys. 106, 472, 503 (1977); B. D. Chang and J. P. Draayer, Phys. Rev. C20, 2387 (1979).

[29] V. Potbhare, Nucl. Phys. A289, 373 (1977).

[30] J. B. French, V. K. B. Kota, A. Pandey, and S. Tomsovic, Ann. Phys. (N.Y.) 181, 235 (1988).

[31] V. K. B. Kota and D. Majumdar, Z. Phys. A 351, 365 (1995); Z. Phys. A 351, 377 (1995).

[32] S. Tomsovic, M. B. Johnson, A. C. Hayes, and J. D.
Bowman, Phys. Rev. C62, 054607 (2000).

[33] J. M. G. Gomez, K. Kar, V. K. B. Kota, R. A. Molina, and J. Retamosa, Phys. Lett. B 567, 251 (2003).

[34] M. Horoi, M. Ghita, and V. Zelevinsky, Phys. Rev. C69, 041307(R) (2004); M. Horoi, J. Kaiser, and V. Zelevinsky, Phys. Rev. C67, 054309 (2003).

[35] W. Li et al., Europhys. Lett., 64, 750 (2003).

[36] N. D. Chavda, V. Potbhare, V. K. B. Kota, Phys. Lett., A 326, 47 (2004).

[37] V. K. B. Kota, Phys. Rev. C71, 041304(R) (2005).

[38] D. Angom and V. K. B. Kota, Phys. Rev. A71, 042504 (2005).

[39] Y. M. Zhao, A. Arima, N. Yoshida, K. Ogawa, N. Yoshinaga, and V. K. B. Kota, Phys. Rev. C72, 064314 (2005).

[40] K. F. Ratcliff, Phys. Rev. C3, 117 (1971).

[41] J. B. French, in Dynamic Structure of Nuclear States, ed. D. J. Rowe et al. (Univ. of Toronto Press, Toronto, 1972), p.154.

[42] B. J. Dalton, W. J. Baldridge, and J. P. Vary, Phys. Rev. C20, 1908 (1979).

[43] J. B. French, Nucl. Phys. A396, 87c (1983).

[44] J. P. Draayer and G. Rosensteel Phys. Lett. 124B, 281 (1983); G. Rosensteel and J. P. Draayer, Nucl. Phys. A436, 445 (1985).

[45] S. Sarkar, K. Kar, and V. K. B. Kota, Phys. Rev. C36, 2700 (1987).

[46] T. Otsuka et al., Phys. Rev. Lett. 87, 082502 (2001).

[47] M. Dufour and A. P. Zuker, Phys. Rev. C54, 1641 (1996).

[48] J. C. Parikh, Group Symmetries in Nuclear Structure (Plenum, New York) (1978).

[49] M. Hjorth-Jensen, T. T. S. Kuo, and E. Osnes, Phys. Rep. 261, 125 (1995).

[50] K. D. Sviratcheva, A. I. Georgieva, and J. P. Draayer, Phys. Rev. C72 054302 (2005).

[51] J. P. Draayer and G. Rosensteel, Phys. Lett. 125B, 237 (1983).

[52] C. Bahri, J. P. Draayer, O. Castanos, and G. Rosensteel Phys. Lett. B 234, 430 (1990)

[53] J. Retamosa, E. Caurier, F. Nowacki, and A. Poves, Phys. Rev. C55, 1266 (1997).

[54] B.D. Chang, Nucl. Phys. A304, 127 (1978).

[55] J. B. French, Phys. Lett. 26B, 75 (1967).

[56] B. D. Chang, J. P. Draayer, and S. S. M. Wong, Comput. Phys. Commun. 28, 41 (1982).

[57] J. B. French, in Isospin in Nuclear Physics, ed. D. H. Wilkinson (North Holland,Amsterdam, 1969), p.259 